\title{
ON ORTHOGONAL POLYNOMIALS SPANNING A NON-STANDARD FLAG
}

\author{
DAVID GÓMEZ-ULLATE, NIKY KAMRAN, AND ROBERT MILSON
}

\begin{abstract}
We survey some recent developments in the theory of orthogonal polynomials defined by differential equations. The key finding is that there exist orthogonal polynomials defined by 2 nd order differential equations that fall outside the classical families of Jacobi, Laguerre, and Hermite polynomials. Unlike the classical families, these new examples, called exceptional orthogonal polynomials, feature non-standard polynomial flags; the lowest degree polynomial has degree $m>0$. In this paper we review the classification of codimension $m=1$ exceptional polynomials, and give a novel, compact proof of the fundamental classification theorem for codimension 1 polynomial flags. As well, we describe the mechanism or rational factorizations of 2 nd order operators as the analogue of the Darboux transformation in this context. We finish with the example of higher codimension generalization of Jacobi polynomials and perform the complete analysis of parameter values for which these families have non-singular weights.
\end{abstract}

\section{INTRODUCTION}

Even though the orthogonal polynomials of Hermite, Laguerre and Jacobi arose from various applications in applied mathematics and physics, these three families now serve as the foundational examples of orthogonal polynomial theory. As such, these classical examples admit many interesting and fruitful generalizations.

A key property of classical orthogonal polynomials is the fact that they can be defined by means of a Sturm-Liouville problem. One of the foundational results in this area a Theorem of Bochner [5] which states that if an infinite sequence of polynomials of degree $0,1,2, \ldots$ satisfies a second order eigenvalue equation of the form

$$
p(x) y^{\prime \prime}+q(x) y^{\prime}+r(x) y=\lambda y
$$

2000 Mathematics Subject Classification. Primary: 34L10, 42C05; Secondary: $33 \mathrm{C} 45,34 \mathrm{~B} 24$.

Key words and phrases. Orthogonal polynomials; Sturm-Liouville problems; Exceptional polynomial subspaces. 
then $p(x), q(x)$ and $r(x)$ must be polynomials of degree at most 2,1 and 0 respectively. In addition, if the eigenpolynomial sequence is $L^{2}$ orthogonal relative to a measure with finite moments, then it has to be, up to an affine transformation of $x$, one of the three classical classical families listed above [1, 29, 12, 27, 26]

Implicit in the above definition of classical polynomials is the assumption that orthogonal polynomials form a basis for the standard polynomial flag $\mathcal{P}_{0} \subset \mathcal{P}_{1} \subset \mathcal{P}_{2} \subset \ldots$, where $\mathcal{P}_{n}=\operatorname{span}\left\{1, x, x^{2}, \ldots, x^{n}\right\}$. In a pair of recent papers [20, 21], we showed that there exist complete orthogonal polynomial systems defined by Sturm-Liouville problems that extend beyond the above classical examples. What distinguishes our hypotheses from those made by Bochner is that the eigenfunction corresponding to the lowest eigenpolynomial of the sequence need not be of degree zero, even though the full set of eigenfunctions still forms a basis of the weighted $\mathrm{L}^{2}$ space.

Already some 20 years ago, particular examples of Hermite-like polynomials with non-standard flags were described in the context of supersymmetric quantum mechanics [9, 6]. The last few years have seen a great deal of activity in the area of non-standard flags; the topic now goes under the rubric of exceptional orthogonal polynomials. There are applications to shape-invariant potentials [30], supersymmetric transformations [22], to discrete quantum mechanics [36], mass-dependent potentials [28], and to quasi-exact solvability [42]. As well, there are now examples of orthogonal polynomials that are solutions of 2 nd order equations and that form flags of arbitrarily high codimension [35].

In light of the activity surrounding orthogonal polynomials with nonstandard flags, we hope that it will be useful to summarize some key results and findings, and to supply stand-alone proofs to some key propositions. We note that the adjective "exceptional" was introduced in the context of our investigation of the equivalence problem for polynomial subspaces [20, 7].

\section{Preliminaries}

2.1. Polynomial flags. Let $U_{1} \subset U_{2} \subset \ldots$, where $\operatorname{dim} U_{k}=k$ be a flag of real, finite-dimensional, polynomial subspaces. Let $n_{k}$ denote the degree of $U_{k}$; that is $n_{k}$ is the maximum of the degrees of the polynomials $p \in U_{k}$. Let $\ell_{k}=n_{k}+1-k$ denote the codimension of $U_{k}$ in $\mathcal{P}_{n_{k}}$, where the $n+1$ dimensional polynomial vector spaces

$$
\mathcal{P}_{n}=\operatorname{span}\left\{1, x, x^{2}, \ldots, x^{n}\right\}
$$


make up the standard polynomial flag: $\mathcal{P}_{0} \subset \mathcal{P}_{1} \subset \mathcal{P}_{2} \subset \ldots$ We say that $\left\{p_{k}\right\}_{k=1}^{\infty}$ is a basis of the flag if

$$
U_{k}=\operatorname{span}\left\{p_{1}, \ldots, p_{k}\right\}
$$

Note that no generality is lost if we assume that $\operatorname{deg} p_{k}=n_{k}$.

Definition 2.1. We call $\mathcal{U}=\left\{U_{k}\right\}_{k=1}^{\infty}$ a degree-regular flag if $n_{k}<n_{k+1}$ for all $k$. Equivalently, a flag is degree-regular if it admits a basis $\left\{p_{k}\right\}_{k=1}^{\infty}$ such that $\operatorname{deg} p_{k}<\operatorname{deg} p_{k+1}$ for all $k$.

Proposition 2.1. A polynomial flag is degree-regular if and only if the codimension sequence obeys $\ell_{k} \leq \ell_{k+1}$ for all $k$.

Henceforth, we will always assume that all flags under discussion are degree-regular.

Definition 2.2. We say that the codimension of a polynomial flag is semi-stable if the codimension sequence $\ell_{k}$ admits an upper bound. If so, we call $\ell=\lim _{k} \ell_{k}$ the codimension of the flag. We say that codimension is stable if $\ell_{k}=\ell$ is constant for all $k$.

Likewise, whenever we speak of the codimension of a polynomial flag, we always assume that the flag has a semi-stable codimension.

Let $\mathcal{D}_{2}(\mathcal{U})$ denote the vector space of 2 nd order differential operators with rational real-valued coefficients

$$
T(y)=p(x) y^{\prime \prime}+q(x) y^{\prime}+r(x) y, \quad p, q, r \in \mathbb{R}(x)
$$

that preserve $\mathcal{U}$; i.e., $T\left(U_{k}\right) \subset U_{k}$ for all $k$. If $p, q, r$ are polynomials, we call $T$ a polynomial operator. Equivalently, an operator is nonpolynomial if and only if it has a pole.

Definition 2.3. We say that $\mathcal{U}$ is imprimitive if it admits a common factor; i.e., $\mathcal{U}$ is spanned by $\left\{q p_{k}\right\}$ where $q(x)$ is a polynomial of degree $\geq 1$. Otherwise, we call $\mathcal{U}$ primitive.

At this juncture, it is important to state the following two Propositions.

Proposition 2.2. Let $T(y)=p y^{\prime \prime}+q y^{\prime}+r y$ be a differential operator such that

$$
T\left(y_{i}\right)=g_{i}, i=1,2,3,
$$

where $y_{i}, g_{i}$ are polynomials with $y_{1}, y_{2}, y_{3}$ linearly independent. Then, $p, q, r$ are rational functions with the Wronskian

$$
W\left(y_{1}, y_{2}, y_{3}\right)=\operatorname{det}\left(\begin{array}{ccc}
y_{1}^{\prime \prime} & y_{1}^{\prime} & y_{1} \\
y_{2}^{\prime \prime} & y_{2}^{\prime} & y_{2} \\
y_{3}^{\prime \prime} & y_{3}^{\prime} & y_{3}
\end{array}\right)
$$

in the denominator. 
Proof. It suffices to apply Cramer's rule to solve the linear system

$$
\left(\begin{array}{lll}
y_{1}^{\prime \prime} & y_{1}^{\prime} & y_{1} \\
y_{2}^{\prime \prime} & y_{2}^{\prime} & y_{2} \\
y_{3}^{\prime \prime} & y_{3}^{\prime} & y_{3}
\end{array}\right)\left(\begin{array}{c}
p \\
q \\
r
\end{array}\right)=\left(\begin{array}{l}
g_{1} \\
g_{2} \\
g_{3}
\end{array}\right)
$$

We should also note that there is a natural linear isomorphism between the space $\mathcal{D}_{2}(\mathcal{U})$ of a flag $\mathcal{U}$ spanned by $\left\{p_{k}\right\}_{k=1}^{\infty}$ and the space $\mathcal{D}_{2}(\tilde{\mathcal{U}})$ of the imprimitive flag $\tilde{\mathcal{U}}$ spanned by $\left\{q p_{k}\right\}_{k=1}^{\infty}$.

Proposition 2.3. Let $T(y)$ be an operator that preserves $\mathcal{U}$. Then, the gauge-equivalent operator $\tilde{T}=q T q^{-1}$ preserves $\tilde{\mathcal{U}}$.

Proposition 2.2 makes clear why we restrict our definition of $\mathcal{D}_{2}$ to operators with rational coefficients. Proposition 2.3 explains the need for primitive flags; these are the canonical representatives for the equivalence relation under gauge transformations.

Definition 2.4. Let $\mathcal{U}$ be a polynomial flag of semi-stable codimension. We say that $\mathcal{U}$ is an exceptional flag if $\mathcal{U}$ is primitive and if $\mathcal{D}_{2}(\mathcal{U})$ does not preserve a polynomial flag of smaller codimension.

Here are some examples to illustrate the above definitions.

Example 2.1. The codimension 1 flag spanned by $1, x^{2}, x^{3}, \ldots$, is exceptional because the non-polynomial operator

$$
T(y)=y^{\prime \prime}-2 y^{\prime} / x
$$

preserves the flag. Since $\ell_{1}=0, \ell_{k}=1, k \geq 2$, the codimension of this flag is not stable, but only semi-stable.

Example 2.2. By contrast, the flag spanned by $x+1, x^{2}, x^{3}, \ldots$ has a stable codimension $\ell=1$. This flag is exceptional because it is preserved by the non-polynomial operator

$$
T(y)=y^{\prime \prime}-2(1+1 / x) y^{\prime}+(2 / x) y .
$$

Example 2.3. Let $h_{k}(x)$ denote the degree $k$ Hermite polynomial. The codimension 1 flag spanned by $h_{1}, h_{2}, h_{3}, \ldots$ is not exceptional. The flag is preserved by the operator $T(y)=y^{\prime \prime}-x y^{\prime}$, but this operator also preserves the standard, codimension zero, polynomial flag.

Example 2.4. The codimension 1 flag spanned by $x, x^{2}, x^{3}, \ldots$ is not exceptional because $x$ is a common factor. The flag is preserved by the operator

$$
\tilde{T}(y)=y^{\prime \prime}-2 y^{\prime} / x+2 y / x^{2}
$$


However, observe that $\tilde{T}=x T x^{-1}$ where $T(y)=y^{\prime \prime}$ is an operator that preserves the standard, codimension zero, polynomial flag.

Example 2.5. Let

$$
y_{2 k-1}=x^{2 k-1}-(2 k-1) x, y_{2 k}=x^{2 k}-k x^{2}, \quad k=2,3,4, \ldots
$$

Consider the codimension-2 flag spanned by $1, y_{3}, y_{4}, y_{5}, y_{6}, \ldots$ The degree sequence of the flag is $0,3,4,5, \ldots$; therefore the codimension is not stable, but merely semi-stable. The flag is preserved by the following operators [17]:

$$
\begin{aligned}
& T_{3}(y)=\left(x^{2}-1\right) y^{\prime \prime}-2 x y^{\prime}, \\
& T_{2}(y)=x y^{\prime \prime}-2\left(1+2 /\left(x^{2}-1\right)\right) y^{\prime}, \\
& T_{1}(y)=y^{\prime \prime}+x\left(1-4 /\left(x^{2}-1\right)\right) y^{\prime} .
\end{aligned}
$$

The flag is exceptional, because $T_{1}$ and $T_{2}$ do not preserve the standard, codimension zero flag. These operators cannot preserve a codimension 1 flag, because, as will be shown in Lemma 3.2, an operator that preserves a codimension 1 flag can have at most 1 pole.

\subsection{Orthogonal polynomials.}

Definition 2.5. We will say that a 2 nd order operator $T(y)$ is exactly solvable by polynomials if the eigenvalue equation

$$
T(y)=\lambda y \text {. }
$$

has infinitely many eigenpolynomial solutions $y=y_{j}$ with

$$
\operatorname{deg} y_{j}<\operatorname{deg} y_{j+1}, \quad j=1,2, \ldots
$$

Let $I=\left(x_{1}, x_{2}\right)$ be an open interval (bounded, unbounded, or semibounded) and let $W(x) d x$ be a positive measure on $I$ with finite moments of all orders. We say that a sequence of real polynomials $\left\{y_{j}\right\}_{j=1}^{\infty}$ forms an orthogonal polynomial system (OPS for short) if the polynomials constitute an orthogonal basis of the Hilbert space $\mathrm{L}^{2}(I, W d x)$. If the codimension of the corresponding flag is stable, or semi-stable, we will say that the OPS has codimension $m$.

The following definition encapsulates the notion of a system of orthogonal polynomials defined by a second-order differential equation. Consider the boundary value problem

$$
\begin{aligned}
& -\left(P y^{\prime}\right)^{\prime}+R y=\lambda W y \\
& \lim _{x \rightarrow x_{i}^{ \pm}}\left(P y^{\prime} u-P u^{\prime} y\right)(x)=0, \quad i=1,2,
\end{aligned}
$$


where $P(x), W(x)>0$ on the interval $I=\left(x_{1}, x_{2}\right)$, and where $u(x)$ is a fixed polynomial solution of (9). We speak of a polynomial SturmLiouville problem (PSLP) if the resulting spectral problem is selfadjoint, pure-point and if all eigenfunctions are polynomial.

If the eigenpolynomials span the standard flag, then we recover the classical orthogonal polynomials, the totality of which is covered by Bochner's theorem. If the solution flag has a codimension $m>0$, Bochner's theorem no longer applies and we encounter a generalized class of polynomials; we name these exceptional, or $X_{m}$ polynomials.

Given a PSLP, the operator

$$
T(y)=W^{-1}\left(P y^{\prime}\right)^{\prime}-W^{-1} R y
$$

is exactly solvable by polynomials. Letting $p(x), q(x), r(x)$ be the rational coefficients of $T(y)$ as in (3), we have

$$
\begin{aligned}
P(x) & =\exp \left(\int^{x} q / p d x\right), \\
W(x) & =(P / p)(x), \\
R(x) & =-(r W)(x),
\end{aligned}
$$

Hence, for a PSLP, $P(x), R(x), W(x)$ belong to the quasi-rational class [15], meaning that their logarithmic derivative is a rational function.

Conversely, given an operator $T(y)$ exactly solvable by polynomials and an interval $I=\left(x_{1}, x_{2}\right)$ we formulate a PLSP (9) by employing (11) -(13) as definitions, and by adjoining the following assumptions:

(PSLP1) $P(x), W(x)$ are continuous and positive on $I$

(PSLP2) $W d x$ has finite moments: $\int_{I} x^{n} W(x) d x<\infty, \quad n \geq 0$

(PSLP3) $\lim _{x \rightarrow x_{i}} P(x) x^{n}=0, \quad i=1,2, \quad n \geq 0$

(PSLP4) the eigenpolynomials of $T(y)$ are dense in $\mathrm{L}^{2}(I, W d x)$.

These definitions and assumptions (PSLP1), (PSLP2) imply Green's formula:

$$
\int_{x_{1}}^{x_{2}} T(f) g W d x-\int_{x_{1}}^{x_{1}} T(g) f W d x=\left.P\left(f^{\prime} g-f g^{\prime}\right)\right|_{x_{1}} ^{x_{2}}
$$

By (PSLP3) if $f(x), g(x)$ are polynomials, then the right-hand side is zero. If $f$ and $g$ are eigenpolynomials of $T(y)$ with unequal eigenvalues, then necessarily, they are orthogonal in $\mathrm{L}^{2}(I, W d x)$. Finally, by (PSLP4) the eigenpolynomials of $T(y)$ are complete in $\mathrm{L}^{2}(I, W d x)$, and hence satisfy the definition of an OPS. 


\section{Codimension 1 Flags}

The key result in this paper is the following. An analogous theorem for polynomial subspaces, rather than flags, was proved in [20].

Theorem 3.1. Up to affine transformations, the flag spanned by $\{x+$ $\left.1, x^{2}, x^{3}, \ldots\right\}$ is the unique stable codimension 1 exceptional flag.

The proof proceeds by means of two lemmas.

Lemma 3.1. A primitive, codimension 1 polynomial flag is exceptional if and only if $\mathcal{D}_{2}$ includes a non-polynomial operator.

Proof. It's clear that a non-polynomial operator cannot preserve the standard polynomial flag. The standard flag is the unique codimension zero flag. Therefore, if a non-polynomial operator preserves a primitive, codimension 1 flag, that flag must be exceptional.

Let us prove the converse. Let $T(y)$ be a polynomial operator that preserves a polynomial flag. Consider the degree homogeneous decomposition

$$
T(y)=\sum_{d=-2}^{N} T_{d}(y)
$$

where

$$
T_{d}(y)=x^{d}\left(\alpha_{d} x^{2} y^{\prime \prime}+\beta_{d} x y^{\prime}+\gamma_{d} y\right)
$$

and where $T_{N}$ is non-zero. Since the operator is polynomial, we must have $\beta_{-2}=\gamma_{-2}=\gamma_{-1}=0$. Also note that

$$
T_{d}\left(x^{j}\right)=\left(j(j-1) \alpha_{d}+\beta_{d} j+\gamma_{d}\right) x^{j+d} .
$$

Let

$$
y_{k}(x)=x^{n_{k}}+\text { lower deg. terms, } k=1,2, \ldots
$$

be a basis of the flag. If $N>0$, then the leading term $T_{N}(y)$ raises degree, and hence

$$
T_{N}\left(x^{n_{k}}\right)=0
$$

for all $k$. By (17), $T_{N}(y)$ annihilates at most 2 distinct monomials, a contradiction. Therefore, $N \leq 0$, and we conclude that $T(y)$ is a Bochner-type operator (1). However, such an operator preserves the standard polynomial flag. Therefore, if $\mathcal{U}$ is exceptional, then there is at least one operator in $\mathcal{D}_{2}(\mathcal{U})$ that doesn't preserve the standard flag. By the above argument, this operator must be non-polynomial.

Lemma 3.2. Let $T(y)$ be a non-polynomial operator that leaves invariant a codimension 1 polynomial subspace $U \subset \mathcal{P}_{n}$. If $n \geq 5$, then $T(y)$ 
has exactly one pole. Furthermore, up to a translation in $x$, a basis of $U$ has one of the following three forms:

$$
\begin{aligned}
& x, x^{2}, x^{3}, \ldots, x^{n} \\
& 1+a x, x^{2}, x^{3}, \ldots, x^{n} \\
& 1+a_{1} x^{2}, x+a_{2} x^{2}, x^{3}, \ldots, x^{n}
\end{aligned}
$$

Proof. By applying a translation if necessary, there is no loss of generality in assuming that $x=0$ is a pole of $T(y)$. Since the codimension is 1 , the subspace admits an order-reduced basis of the form

$$
\begin{aligned}
y_{j} & =x^{j-1}+a_{j-1} x^{\nu}, & j & =1, \ldots, \nu \\
y_{j} & =x^{j}, & j & =\nu+1 \ldots n,
\end{aligned}
$$

where $0 \leq \nu \leq n$. The matrix representation of this basis is a $n \times n$ matrix in row-reduced echelon form. This matrix has $n$ pivots and 1 gap in position $\nu$.

Our first claim is that $\nu \leq 2$. Suppose not. Then

$$
W\left(y_{1}, y_{2}, y_{3}\right)=W\left(1, x, x^{2}\right)+\text { higher degree terms }=2+O(x) .
$$

By assumption, $T\left(y_{i}\right)$ is a polynomial. Hence, Proposition 2.2 implies that $x=0$ is not a pole of the operator, a contradiction.

Having established that $\nu \leq 2$, we observe that $x^{3}, x^{4}, x^{5} \in U$. The Wronskian of these monomials is a multiple of $x^{9}$. Therefore, by Proposition 2.2, $x=0$ is the unique pole.

We are now ready to give the proof of Theorem 3.1. Let $\mathcal{U}$ be an exceptional polynomial flag with stable codimension 1. By Lemma 3.1, there exists a non-polynomial operator $T(y)$ that preserves the flag. By Lemma 3.2, this operator has a unique pole. Without loss of generality we assume that $x=0$ is the unique pole. We rule out possibility (18), because if this holds for even one $U_{k}, k \geq 5$, then it must hold for all $k$. This would imply that $x, x^{2}, x^{3}, \ldots$ is a basis of the flag - a violation of the primitivity assumption.

Let us rule out possibility (201). Let $T(y)$ be a non-polynomial operator that preserves the flag. Since $x=0$ is the unique pole, we can decompose the operator into degree-homogeneous terms

$$
T(y)=\sum_{d=N_{1}}^{N_{2}} T_{d}(y)
$$

where $T_{d}(y)$ has the form shown in (16), and where $T_{N_{1}}$ and $T_{N_{2}}$ are non-zero. By the argument used in the proof of Lemma 3.1. we must 
have $N_{2} \leq 0$. By assumption, $T\left(1+a_{1} x^{2}\right), T\left(x+a_{2} x^{2}\right), T\left(x^{3}\right)$ are polynomials. However, if $N_{1} \leq-4$, then this condition requires that

$$
T_{N_{1}}(1)=T_{N_{1}}(x)=T_{N_{1}}\left(x^{3}\right)=0,
$$

which means that $T_{N_{1}}$ is zero - a contradiction. If $N_{1}=-3$, then $T_{-3}$ annihilates $1, x, x^{5}$, a contradiction. If $N_{1}=-2$, then $T_{-2}$ annihilates $1, x, x^{4}$, another contradiction. Similarly, if $N_{1}=-1$, then $T_{-1}$ annihilates 1. Hence $T_{-1}(y)=\alpha_{-1} x y^{\prime \prime}+\beta_{-1} y^{\prime}$. This means that there is no pole - a contradiction. Therefore, $N_{1}=N_{2}=0$, but that means that $T(y)$ is a polynomial operator - again, a contradiction. This rules out possibility (20).

This leaves (19) as the only possibility. Since we assume that the codimension is stable, $\operatorname{deg}(1+a x)=1$ and hence $a \neq 0$. We scale $x$ to transform $1+a x$ to $x+1$. This concludes the proof of the main theorem.

3.1. $X_{1}$ polynomials. The above theorem explains the origin of the adjective "exceptional" and leads directly to a more general class of orthogonal polynomials — outside the class described by Bochner's theorem. Two families of orthogonal polynomials arise naturally when we consider codimension 1 flags. We describe these $X_{1}$ polynomials below.

In order to construct codimension 1 polynomial systems, we must consider $\mathcal{D}_{2}$ of the flag spanned by $x+1, x^{2}, x^{3}, \ldots$.

Proposition 3.1. The most general 2nd order operator that preserves the flag $\left\{x+1, x^{2}, x^{3}, \ldots\right\}$ has the form

$$
T(y)=\left(k_{2} x^{2}+k_{1} x+k_{0}\right) y^{\prime \prime}-(x+1)\left(k_{1}+\frac{2 k_{0}}{x}\right) y^{\prime}+\left(k_{1}+\frac{2 k_{0}}{x}\right) y,
$$

where $k_{2}, k_{1}, k_{0}$ are real constants.

See [19, Proposition 4.10] for the proof.

Thus to obtain $X_{1}$ orthogonal polynomials it suffices to determine all possible values of $k_{2}, k_{1}, k_{0}$ for which $P(x), W(x)$ as given by (11) (12) satisfy the conditions of a PSLP. This analysis is performed in 21]. In summary, non-singular weights arise only for the case where $k_{2} x^{2}+k_{1} x+k_{0}$ either has two distinct real roots or if $k_{2}=0, k_{1} \neq 0$. The first case leads to the Jacobi $X_{1}$ polynomials; the second leads to the $X_{1}$ Laguerre polynomials. In both cases, the polynomial flags span a dense subspace of the respective Hilbert space [21, Proposition 3.1, Proposition 3.3]. We summarize the key properties of these two families below. 
3.2. $X_{1}$-Jacobi polynomials. Let $\alpha \neq \beta$ be real parameters such that

$$
\alpha>-1, \quad \beta>-1, \quad \operatorname{sgn} \alpha=\operatorname{sgn} \beta .
$$

Set

$$
a=\frac{1}{2}(\beta-\alpha), \quad b=\frac{\beta+\alpha}{\beta-\alpha}, \quad c=b+1 / a .
$$

Note that, with the above assumptions, we have $|b|>1$. Now let

$$
u_{1}=x-c, \quad u_{i}=(x-b)^{i}, \quad i \geq 2 .
$$

Define the measure $d \hat{\mu}_{\alpha, \beta}=\hat{W}_{\alpha, \beta} d x$ where

$$
\hat{W}_{\alpha, \beta}=\frac{(1-x)^{\alpha}(1+x)^{\beta}}{(x-b)^{2}}, \quad x \in(-1,1) .
$$

Since $\hat{W}_{\alpha, \beta}>0$ for $-1<x<1$, the scalar product

$$
(f, g)_{\alpha, \beta}:=\int_{-1}^{1} f(x) g(x) d \hat{\mu}_{\alpha, \beta}
$$

is positive definite. Also note that the above measure has finite moments of all orders.

We now define the $X_{1}$-Jacobi polynomials $\hat{P}_{n}^{(\alpha, \beta)}, n=1,2, \ldots$ as the sequence obtained by orthogonalization of the flag spanned by $u_{1}, u_{2}, u_{3}, \ldots$ with respect to the scalar product (26), and by imposing the normalization condition 1

$$
\hat{P}_{n}^{(\alpha, \beta)}(1)=n\left(\begin{array}{c}
\alpha+n-1 \\
n
\end{array}\right) .
$$

From their definition it is obvious that $\operatorname{deg} \hat{P}_{n}^{(\alpha, \beta)}=n$. However, as opposed to the ordinary Jacobi polynomials, the sequence starts with a degree one polynomial.

Next, define the 2nd order operator

$$
T_{\alpha, \beta}(y)=\left(x^{2}-1\right) y^{\prime \prime}+2 a\left(\frac{1-b x}{b-x}\right)\left((x-c) y^{\prime}-y\right),
$$

An elementary calculation shows that this operator preserves the flag $\left\{u_{i}\right\}$ as defined above. Indeed, the flag $\left\{u_{i}\right\}$ and the operator (28) are obtained from the flag $x+1, x^{2}, x^{3}, \ldots$ and from the operator (21) via the following specialization and affine transformation

$$
k_{2}=1, \quad k_{1}=-2 a b, \quad k_{0}=\left(1-b^{2}\right) a, \quad x \rightarrow-a(x-b) .
$$

\footnotetext{
${ }^{1}$ This convention differs from the one adopted in 21. The change is made to conform with the convention adopted below for the generalized $X_{m}$ Jacobi polynomials.
} 
Multiplying both sides of the equation

$$
-T_{\alpha, \beta}(y)=\lambda y
$$

by $\hat{W}_{\alpha, \beta}$ leads to the following PSLP:

$$
\begin{aligned}
& \left(\left(1-x^{2}\right) \hat{W}_{\alpha, \beta} y^{\prime}\right)^{\prime}+2 a\left(\frac{1-b x}{b-x}\right) \hat{W}_{\alpha, \beta} y=\lambda \hat{W}_{\alpha, \beta} y, \\
& \lim _{x \rightarrow 1^{-}}(1-x)^{\alpha+1}\left((x-c) y^{\prime}-y\right)=0, \\
& \lim _{x \rightarrow-1^{+}}(1+x)^{\beta+1}\left((x-c) y^{\prime}-y\right)=0 .
\end{aligned}
$$

The boundary conditions select polynomial solutions. The self-adjoint form of (30) ensures that the solutions are orthogonal relative to $d \hat{\mu}_{\alpha, \beta}$. Therefore, the $X_{1}$ Jacobi polynomials can also be described as polynomial solutions, $y=\hat{P}_{n}^{(\alpha, \beta)}(x)$, of the following 2 nd order equation:

$$
T_{\alpha, \beta}(y)=(n-1)(\alpha+\beta+n) y \text {. }
$$

3.3. $X_{1}$-Laguerre polynomials. For $k>0$, set

$$
v_{1}=x+k+1, \quad v_{i}=(x+k)^{i}, \quad i \geq 2
$$

Define the measure $d \hat{\mu}_{k}=\hat{W}_{k} d x$ where

$$
\hat{W}_{k}=\frac{e^{-x} x^{k}}{(x+k)^{2}}, \quad x \in(0, \infty)
$$

and observe that $\hat{W}_{k}>0$ on the domain in question. Therefore, the following inner product is positive definite:

$$
(f, g)_{k}:=\int_{0}^{\infty} f(x) g(x) d \hat{\mu}_{k},
$$

Also note that the above measure has finite moments of all orders.

We define the $X_{1}$-Laguerre polynomials $\hat{L}_{k, i}, i=1,2,3, \ldots$ as the sequence obtained by orthogonalization of the flag spanned by $v_{1}, v_{2}, v_{3}, \ldots$ with respect to the scalar product (36) and subject to the normalization condition

$$
\hat{L}_{k, n}(x)=\frac{(-1)^{n} x^{n}}{(n-1) !}+\text { lower order terms } \quad n \geq 1 .
$$

Again, since we are orthogonalizing a non-standard flag, the $X_{1}$-Laguerre polynomial sequence starts with a polynomial of degree 1 , rather than a polynomial of degree 0 .

Define the operator

$$
T_{k}(y)=-x y^{\prime \prime}+\left(\frac{x-k}{x+k}\right)\left((x+k+1) y^{\prime}-y\right)
$$


and note that this operator leaves invariant the flag spanned by the $v_{i}$. Indeed the flag $\left\{v_{i}\right\}$ and (38) are obtained from the flag $x+1, x^{2}, \ldots$ and (21) via the specializations and an affine transformation shown below:

$$
k_{2}=0, k_{1}=-1, k_{0}=k, \quad x \mapsto x+k
$$

The corresponding PSLP takes the form

$$
\begin{gathered}
\left(x \hat{W}_{k} y^{\prime}\right)^{\prime}+\left(\frac{x-k}{x+k}\right) \hat{W}_{k} y=\lambda y, \\
\lim _{x \rightarrow 0^{+}} x^{k+1}\left((x+k+1) y^{\prime}-y\right)=0, \\
\lim _{x \rightarrow \infty} e^{-x}\left((x+k+1) y^{\prime}-y\right)=0 .
\end{gathered}
$$

As before, the boundary conditions select polynomial solutions, while the self-adjoint form of (40) ensures that these solutions are orthogonal relative to $d \hat{\mu}_{k}$. Therefore, the $X_{1}$ Laguerre polynomials can also be defined as polynomial solutions, $y=\hat{L}_{k, n}$, of the following 2 nd order equation:

$$
T_{k}(y)=(n-1) y \text {. }
$$

Having introduced the $X_{1}$ Jacobi and Laguerre polynomials, we are able to state the following corollary of Theorem [3.1. The proof is found in [21].

Theorem 3.2. The $X_{1}$-Jacobi polynomials and the $X_{1}$ Laguerre polynomials are the unique orthogonal polynomial systems defined by a stable codimension-1 PSLP.

\section{Higher CODimension FLAGS}

Even though the first examples of exceptional orthogonal polynomials involve codimension-1 flags, recently announced examples [35] are proof that exceptional orthogonal polynomials can span flags of arbitrarily high codimension. Another important development is the recent proof [30] that the codimension-1 families can be related to the classical orthogonal polynomials by means of a Darboux transformation. In a follow-up publication [22], it was shown that the new higher codimension examples are systematically derivable by means of algebraic Darboux transformations [17].

A closely related development involves the notion of shape-invariance [13], a methodology related to the study of exactly solvable potentials. The close connection between solvable potentials and orthogonal polynomials is well recognized; consider the relationship between the harmonic oscillator and Hermite polynomials, for example. 
In the orthogonal polynomial context, we have to factorize general second order operators, not just Schrödinger operators. The shapeinvariance property of the classical potentials manifests as the Rodrigues' formula for the corresponding polynomials. It has been pointed out that all the potentials related to exceptional orthogonal polynomials exhibit the shape-invariance property [30, 38], and that therefore, like their classical counterparts, exceptional orthogonal polynomials have a Rodrigues' formula. This phenomenon was studied and explained in [22], where it was shown that the shape-invariance property of the $X_{m}$ (exceptional, codimension $m$ ) polynomials follows from the permutability property of higher-order Darboux transformations.

4.1. The Darboux transformation. In the remainder of this section, we review the some key definitions and results related to Darboux transformations and shape-invariance, and then illustrate these ideas with the example of $X_{m}$ Jacobi polynomials [39].

Consider the differential operators:

$$
\begin{aligned}
& T(y)=p y^{\prime \prime}+q y^{\prime}+r y \\
& A(y)=b\left(y^{\prime}-w y\right), \quad B(y)=\hat{b}\left(y^{\prime}-\hat{w} y\right),
\end{aligned}
$$

where $p, q, r, b, w, \hat{b}, \hat{w}$ are rational functions.

Definition 4.1. We speak of a rational factorization if there exists a constant $\lambda_{0}$ such that

$$
T=B A+\lambda_{0}
$$

If the above equation holds, we call

$$
\hat{T}=A B+\lambda_{0}
$$

the partner operator. We call

$$
\phi(x)=\exp \int^{x} w d x, \quad w=\phi^{\prime} / \phi
$$

a quasi-rational factorization eigenfunction and $b(x)$ the factorization gauge.

The reason for the above terminology is as follows. By (46),

$$
T(\phi)=\lambda_{0} \phi
$$

hence the term factorization eigenfunction. Next, consider two factorization gauges $b_{1}(x), b_{2}(x)$ and let $\hat{T}_{1}(y), \hat{T}_{2}(y)$ be the corresponding partner operators. Then,

$$
\hat{T}_{2}=\mu^{-1} \hat{T}_{1} \mu, \quad \text { where } \mu(x)=b_{1}(x) / b_{2}(x) .
$$


Therefore, the choice of $b(x)$ determines the gauge of the partner operator. This is why we refer to $b(x)$ as the factorization gauge. Also, note that in [17] the above construction was referred to as an algebraic Darboux transformation. However, in light of the recently recognized role played by operators with rational coefficients, the term rational factorization seems to be preferable.

Proposition 4.1. Let $T(y)$ be a 2nd order operator exactly solvable by polynomials, and let $\phi(x)$ be a quasi-rational factorization eigenfunction with eigenvalue $\lambda_{0}$. Then, there exists a rational factorization (46) such that the partner operator is also exactly solvable by polynomials, and such that the partner flag is primitive (no common factors).

Proof. Let $w(x)=\phi^{\prime}(x) / \phi(x)$ and let $b(x)$ be an as yet unspecified rational function. Set

$$
\begin{aligned}
& \hat{w}=-w-q / p+b^{\prime} / b, \\
& \hat{b}=p / b,
\end{aligned}
$$

and let $A(y), B(y)$ be as shown in in (45). An elementary calculation shows that (46) holds. Let $y_{1}, y_{2}, \ldots$ be a degree-regular basis of the eigenpolynomials of $T(y)$. We require that the flag spanned by $A\left(y_{j}\right)$ be polynomial and primitive (no common factors). Observe that if we take $b(x)$ to be the reduced denominator of $w(x)$, then $A\left(y_{j}\right)$ is a polynomial for all $j$. However, this does not guarantee that $A\left(y_{j}\right)$ is free of a common factor. That is a stronger condition, one that fixes $b(x)$ up to a choice of scalar multiple. Finally, the intertwining relation

$$
\hat{T} A=A T
$$

implies that the $A\left(y_{j}\right)$ are eigenpolynomials of the partner $\hat{T}$.

Finally, let us derive the formula for the partner weight function.

Proposition 4.2. Suppose that a PSLP operators $T(y)=p y^{\prime \prime}+q y^{\prime}+r y$ is related to a PSLP operator $\hat{T}(y)=p y^{\prime \prime}+\hat{q} y^{\prime}+\hat{r} y$ by a rational factorization with factorization eigenfunction $\phi(x)$ and factorization gauge $b(x)$, Then the dual factorization gauge, factorization eigenfunction and weight function are given by

$$
\begin{aligned}
& b \hat{b}=p \\
& \hat{W} / \hat{b}=W / b, \\
& \hat{b} \hat{\phi}=1 /(W \phi)
\end{aligned}
$$


Proof. Equation (53) follows immediately from (45) (46). Writing

$$
\hat{T}(y)=p y^{\prime \prime}+\hat{q} y^{\prime}+\hat{r} y,
$$

equation (47) implies that

$$
w+\hat{w}=-q / p+b^{\prime} / b=-\hat{q} / p+\hat{b}^{\prime} / \hat{b} .
$$

Hence,

$$
\hat{q}=q+p^{\prime}-2 p b^{\prime} / b .
$$

From here, (54) follows by equations (11) (12). Equation (55) follows from (48).

The adjoint relation between $A$ and $B$ allows us to compare the $\mathrm{L}^{2}$ norms of the two families.

Proposition 4.3. Let $T(y), \hat{T}(y)$ be PSLP operators related by a rational factorization (46) (47). Let $\left\{y_{j}\right\}$ be the eigenpolynomials of $T(y)$ and let $\hat{y}_{j}=A\left(y_{j}\right)$ be the corresponding partner eigenpolynomials. Then

$$
\int_{x_{1}}^{x_{2}} A\left(y_{j}\right)^{2} \hat{W} d x=\left(\lambda_{0}-\lambda_{j}\right) \int_{x_{1}}^{x_{2}} y_{j}^{2} W d x
$$

where $x_{1}<x_{2}$ are the end points of the Sturm-Liouville problem in question.

Proof. As a consequence of (54), $A$ and $-B$ are formally adjoint relative to the respective measures:

$$
\int_{x_{1}}^{x_{2}} A(f) g \hat{W} d x+\int_{x_{1}}^{x_{2}} B(g) f W d x=\left.(P / b) f g\right|_{x_{1}} ^{x_{2}},
$$

where $P(x)$ is defined by (11) and where $b(x)$ is the factorization gauge. By assumption both $W(x), \hat{W}(x)$ are positive on $\left(x_{1}, x_{2}\right)$. By (54), $\hat{W}=P / b^{2}$. In particular, the numerator $b(x)$ cannot have any zeroes in $\left(x_{1}, x_{2}\right)$. As well, if either $x_{1}, x_{2}$ are finite, we cannot have $b\left(x_{i}\right)=0$, because that would imply that $\hat{W}$ is not square integrable near $x=x_{i}$. Hence $|1 / b(x)|$ is bounded from above on $\left(x_{1}, x_{2}\right)$. Therefore, if $f, g$ are polynomials then the right-hand side of (60) vanishes by (PSLP3). Therefore,

$$
\int_{x_{1}}^{x_{2}} A\left(y_{j}\right)^{2} \hat{W} d x=-\int_{x_{1}}^{x_{2}} B\left(A\left(y_{j}\right)\right) y_{j} W d x=\left(\lambda_{0}-\lambda_{j}\right) \int_{x_{1}}^{x_{2}} y_{j}^{2} W d x
$$


4.2. Shape-invariance. Parallel to the $L^{2}$ spectral theory [8, 40], rational factorizations of a solvable operator $T(y)$ can be categorized as formally state-deleting, formally state-adding, or formally isospectral. The connection between these formal, algebraic Darboux transformations and their $L^{2}$ analogues is discussed in [22]. We speak of a formally state-deleting transformation if the factorization eigenfunction $\phi(x)$ is the lowest degree eigenpolynomial of $T(y)$. We speak of a formally state-adding transformation if the partner factorization eigenfunction

$$
\hat{\phi}(x)=\exp \int^{x} \hat{w} d x
$$

with $\hat{w}$ defined by (50), is a polynomial. We speak of a formally isospectral transformation if neither $\phi$ nor $\hat{\phi}$ are polynomials. Examples of all three types of factorizations will be given below.

State-adding and state-deleting factorizations are dual notions, in the sense that if the factorization of $T$ is state-deleting, then the factorization of $\hat{T}$ is state-adding, and vice versa.

Definition 4.2. Let $\kappa \in K$ be a parameter index set and let

$$
T_{\kappa}(y)=p(x) y^{\prime \prime}+q_{\kappa}(x) y^{\prime}+r_{\kappa}(x) y, \quad \kappa \in K,
$$

be a family of operators that are exactly solvable by polynomials. If this family is closed with respect to the state-deleting transformation, we speak of shape-invariant operators.

To be more precise, let $\pi_{\kappa}(x)=y_{\kappa, 1}(x)$ be be the corresponding eigenpolynomial of lowest degree. Without loss of generality, we assume that the corresponding spectral value is zero, and let

$$
T_{\kappa}=B_{\kappa} A_{\kappa}, \quad A_{\kappa} \pi_{\kappa}=0
$$

be the corresponding factorization. Shape-invariance means that there exists a one-to-one map $h: K \rightarrow K$ and real constants $\lambda_{\kappa}$ such that

$$
T_{h(\kappa)}=A_{\kappa} B_{\kappa}+\lambda_{\kappa} .
$$

In accordance with (11), define

$$
P_{\kappa}(x)=\exp \left(\int^{x} q_{\kappa} / p\right)
$$

Let $b_{\kappa}(x)$ denote the shape-invariant factorization gauge; i.e.;

$$
A_{\kappa}(y)=\left(b_{\kappa} / \pi_{\kappa}\right) \mathcal{W}\left(\pi_{\kappa}, y\right)
$$

where

$$
\mathcal{W}(f, g)=f g^{\prime}-f^{\prime} g
$$


denotes the Wronskian operator. By equation (64),

$$
q_{h(\kappa)}=q_{\kappa}+p^{\prime}-2 p b_{\kappa}^{\prime} / b_{\kappa} .
$$

It follows that

$$
p P_{\kappa} / P_{h(\kappa)}=b_{\kappa}^{2} \text {. }
$$

Below, we will use this necessary condition to derive the factorization gauge of a shape-invariant factorization.

4.3. Jacobi polynomials. The operator

$$
T_{\alpha, \beta}(y):=\left(1-x^{2}\right) y^{\prime \prime}+(\beta-\alpha+(\alpha+\beta+2) x) y^{\prime},
$$

preserves the standard flag, and hence is exactly solvable by polynomials. The classical Jacobi polynomials $P_{n}^{(\alpha, \beta)}(x), \alpha, \beta>-1, n=$ $0,1,2, \ldots$ are the corresponding eigenpolynomials:

$$
T_{\alpha, \beta} P_{n}^{(\alpha, \beta)}=-n(n+\alpha+\beta+1) P_{n}^{(\alpha, \beta)},
$$

subject to the normalization

$$
P_{n}^{(\alpha, \beta)}(1)=\left(\begin{array}{c}
n+\alpha \\
n
\end{array}\right)
$$

The $L^{2}$ orthogonality is relative to the measure $W_{\alpha, \beta}(x) d x$ where

$$
W_{\alpha, \beta}(x)=(1-x)^{\alpha}(1+x)^{\beta}, \quad x \in(-1,1) .
$$

The classical operators are shape-invariant by virtue of the following factorizations:

$$
\begin{aligned}
T_{\alpha, \beta} & =\hat{B}_{\alpha, \beta} \hat{A}_{\alpha, \beta} \\
T_{\alpha+1, \beta+1} & =\hat{A}_{\alpha, \beta} \hat{B}_{\alpha, \beta}+\alpha+\beta+2 \quad \text { where } \\
\hat{B}_{\alpha, \beta}(y) & =\left(1-x^{2}\right) y^{\prime}+(\beta-\alpha+(\alpha+\beta+2) x) y, \\
& =(1-x)^{-\alpha}(1+x)^{-\beta}\left(y(1-x)^{\alpha+1}(1+x)^{\beta+1}\right)^{\prime} \\
\hat{A}_{\alpha, \beta}(y) & =y^{\prime} .
\end{aligned}
$$

As a consequence, $B_{\alpha, \beta}(y)$ acts as a raising operator:

$$
B_{\alpha, \beta} P_{n}^{(\alpha+1, \beta+1)}=-2(n+1) P_{n+1}^{(\alpha, \beta)},
$$

and $A_{\alpha, \beta}(y)$ as a lowering operator:

$$
P_{n}^{(\alpha, \beta) \prime}=\frac{1}{2}(1+\alpha+\beta+n) P_{\alpha+1, \beta+1, n-1} .
$$

The classical Rodrigues' formula, namely

$$
(1-x)^{-\alpha}(1-x)^{-\beta} \frac{d^{n}}{d x^{n}}\left((1-x)^{\alpha+n}(1-x)^{\beta+n}\right)=(-2)^{n} n ! P_{n}^{(\alpha, \beta)}(x)
$$


follows by applying $n$ iterations of the raising operator to the constant function.

The quasi-rational solutions of $T_{\alpha, \beta}(y)=\lambda y$ are known [4, Section $2.2]$ :

$$
\begin{array}{lll}
\phi_{1}(x) & =P_{m}^{(\alpha, \beta)}(x), & \lambda_{0}=-m(1+\alpha+\beta+m) \\
\phi_{2}(x)=(1-x)^{-\alpha}(1+x)^{-\beta} P_{-\alpha,-\beta, m}(x) & \lambda_{0}=(1+m)(\alpha+\beta-m) \\
\phi_{3}(x)=(1-x)^{-\alpha} P_{-\alpha, \beta, m}(x) & \lambda_{0}=(1+\beta+m)(\alpha-m) \\
\phi_{4}(x)=(1-x)^{-\beta} P_{\alpha,-\beta, m}(x), & \lambda_{0}=(1+\alpha+m)(\beta-m)
\end{array}
$$

where $m=0,1,2, \ldots$. The corresponding factorizations were analyzed in [16]. Of these, $\phi_{1}$ with $m=0$ corresponds to a state-deleting transformation and underlies the shape-invariance of the classical Laguerre operator and the corresponding Rodrigues formula. For $m>0$, the $\phi_{1}$ factorization eigenfunctions yield singular operators and hence do not yield novel orthogonal polynomials. The $\phi_{2}$ family results in a state-adding transformation. The resulting flags are semi-stable, like in Example 2.5, see [17] for a discussion. The type $\phi_{3} \phi_{4}$ factorizations result in novel orthogonal polynomials, provided $\alpha, \beta$ satisfy certain inequalities. These families were referred to as the J1, J2 Jacobi polynomials in [37]. The two families are related by the transformations $\alpha \leftrightarrow \beta, x \mapsto-x$. We therefore focus only on the $\phi_{3}$ factorization; no generality is lost.

The derivations that follow depend in an elementary fashion on the following well-known identities of the Jacobi polynomials. We will apply them below without further comment.

$$
\begin{aligned}
& P_{0}^{(\alpha, \beta)}(x)=1, \quad P_{n}^{(\alpha, \beta)}(x)=0, \quad n \leq-1, \\
& P_{n}^{(\alpha, \beta)}(x)=(-1)^{n} P_{n}^{(\alpha, \beta)}(-x), \\
& (x-1) P_{m}^{(\alpha, \beta) \prime}(x)=(\alpha+m) P_{m}^{(\alpha-1, \beta+1)}(x)-\alpha P_{m}^{(\alpha, \beta)}(x) \\
& P_{n}^{(\alpha, \beta) \prime}(x)=\frac{1}{2}(1+\alpha+\beta+n) P_{\alpha+1, \beta+1, n-1}(x) \\
& P_{n}^{(\alpha, \beta-1)}(x)-P_{n}^{(\alpha-1, \beta)}(x)=P_{n-1}^{(\alpha, \beta)}(x)
\end{aligned}
$$

4.4. The $X_{m}$ Jacobi polynomials. Fix an integer $m \geq 1$ and $\alpha, \beta>$ -1 , and set

$$
\xi_{\alpha, \beta, m}=P_{m}^{(-\alpha, \beta)}(x)
$$

Take $\phi_{3}(x)$ as the factorization eigenfunction and take

$$
b(x)=(1-x) \xi_{\alpha, \beta, m}
$$


as the factorization gauge. Applying (45) (50) and the identities (81)(85) , we obtain the following rational factorization of the Jacobi operator (28):

$$
\begin{aligned}
& T_{\alpha, \beta}=B_{\alpha, \beta, m} A_{\alpha, \beta, m}-(m-\alpha)(m+\beta+1) \quad \text { where } \\
& A_{\alpha, \beta, m}(y)=(1-x) \xi_{\alpha, \beta, m} y^{\prime}+(m-\alpha) \xi_{\alpha+1, \beta+1, m} y \\
& B_{\alpha, \beta, m}(y)=\left((1+x) y^{\prime}+(1+\beta) y\right) / \xi_{\alpha, \beta, m}
\end{aligned}
$$

By (55), $\hat{\phi}(x)=(1+x)^{-1-\beta}$ is the dual factorization eigenfunction. Since neither $\phi_{3}(x)$ nor $\hat{\phi}(x)$ is a polynomial, (87) is an example of a formally isospectral factorization. The corresponding partner operator is shown below:

$$
\begin{aligned}
T_{\alpha, \beta, m}= & A_{\alpha+1, \beta-1, m} B_{\alpha+1, \beta-1, m}-(m-\alpha-1)(m+\beta), \\
T_{\alpha, \beta, m}(y)= & T_{\alpha, \beta}(y)-2 \rho_{\alpha+1, \beta-1, m}\left(\left(1-x^{2}\right) y^{\prime}+b(1-x) y\right) \\
& \quad+m(a-b-m+1) y, \quad \text { where } \\
\rho_{\alpha, \beta, m}= & \xi_{\alpha, \beta, m}^{\prime} / \xi_{\alpha, \beta, m} \\
= & \frac{1}{2}(1-\alpha+\beta+m) \xi_{\alpha-1, \beta+1, m-1} / \xi_{\alpha, \beta, m}
\end{aligned}
$$

Based on the above factorization, we define the $X_{m}$, exceptional Jacobi polynomials to be

$$
\begin{gathered}
\hat{P}_{n}^{(\alpha, \beta, m)}=\frac{(-1)^{m+1}}{\alpha+1+j} A_{\alpha+1, \beta-1, m} P_{j}^{(\alpha+1, \beta-1)}, \quad j=n-m \geq 0 \\
=(-1)^{m}\left[\frac{1+\alpha+\beta+j}{2(\alpha+1+j)}(x-1) P_{m}^{(-\alpha-1, \beta-1)} P_{j-1}^{(\alpha+2, \beta)}\right. \\
\left.+\frac{1+\alpha-m}{\alpha+1+j} P_{m}^{(-2-\alpha, \beta)} P_{j}^{(\alpha+1, \beta-1)}\right]
\end{gathered}
$$

By construction, these polynomials satisfy

$$
T_{\alpha, \beta, m} \hat{P}_{n}^{(\alpha, \beta, m)}=-(n-m)(1+\alpha+\beta+n-m) \hat{P}_{n}^{(\alpha, \beta, m)}
$$

With the above definition, the generalized Jacobi polynomials obey the following normalization condition

$$
\hat{P}_{n}^{(\alpha, \beta, m)}(1)=\left(\begin{array}{c}
\alpha+n-m \\
n
\end{array}\right)\left(\begin{array}{l}
n \\
m
\end{array}\right), \quad n \geq m .
$$

Note that the $X_{m}$ operators and polynomials extend the classic family:

$$
\begin{aligned}
& T_{\alpha, \beta, 0}(y)=T_{\alpha, \beta}(y) \\
& \hat{P}_{n}^{(\alpha, \beta, 0)}=P_{n}^{(\alpha, \beta)} .
\end{aligned}
$$


The $L^{2}$ norms of the classical polynomials are given by

$$
\int_{-1}^{1}\left[P_{n}^{(\alpha, \beta)}(x)\right]^{2}(1-x)^{\alpha}(1+x)^{\beta} d x=N_{n}^{\alpha, \beta}
$$

where

$$
N_{n}^{\alpha, \beta}=\frac{2^{\alpha+\beta+1} \Gamma(\alpha+1+n) \Gamma(\beta+1+n)}{n !(\alpha+\beta+2 n+1) \Gamma(\alpha+\beta+n+1)}
$$

By (54), the weight for the $X_{m}$ Jacobi polynomials is given by

$$
\hat{W}_{\alpha, \beta, m}(x)=\frac{(1-x)^{\alpha}(1+x)^{\beta}}{\xi_{\alpha+1, \beta-1, m}(x)^{2}}
$$

In order for $L^{2}$ orthogonality to hold for the generalized polynomials, we restrict $\alpha, \beta$ so that the denominator in the above weight is non-zero for $-1<x<1$. We also want to avoid the degenerate cases where $x= \pm 1$ is a root of $\xi_{\alpha+1, \beta-1, m}$. To ensure that we obtain a codimension $m$ flag, we also demand that $\operatorname{deg} \xi_{\alpha+1, \beta-1, m}=m$. The proof of the following Proposition follows from the analysis in [41, Chapter 6.72].

Proposition 4.4. Suppose that $\alpha, \beta>-1$. Then $\operatorname{deg} \xi_{\alpha+1, \beta-1, m}=m$ and $\xi_{\alpha+1, \beta-1, m}( \pm 1) \neq 0$ if and only if $\beta \neq 0$ and

$$
\alpha, \alpha-\beta-m+1 \notin\{0,1, \ldots, m-1\}
$$

Proposition 4.5. Suppose that the conditions of the preceding Proposition hold. The polynomial $\xi_{\alpha+1, \beta-1, m}(x)$ has no zeros in $(-1,1)$ if and only if $\alpha>m-2$ and

$$
\operatorname{sgn}(\alpha-m+1)=\operatorname{sgn}(\beta) .
$$

This is a good place to compare the above results to the parameter inequalities imposed in [37, 39]. These references impose the condition

$$
\alpha>\beta>m-1 / 2 \text {. }
$$

Unlike Proposition 4.5, this condition fails to describe the most general non-singular weight $\hat{W}_{\alpha, \beta, m}$. Consider the following examples:

$$
\begin{gathered}
\hat{W}_{1 / 3,-1 / 2,2}=288^{2} \frac{(1-x)^{1 / 3}(1+x)^{-1 / 2}}{\left(7 x^{2}+2 x-41\right)^{2}} \\
\hat{W}_{5 / 4,1 / 2,2}=128^{2} \frac{(1-x)^{5 / 4}(1+x)^{1 / 2}}{\left(5 x^{2}-14 x+29\right)^{2}}
\end{gathered}
$$

Neither of the above examples satisfy the parameter inequalities of [37, 39], but both weights are non-singular on $(-1,1)$ and have finite 
moments of all orders. On the other hand, the parameter values $m=$ $2, \alpha=3 / 2, \beta=1 / 2$ give

$$
\begin{aligned}
& \hat{W}_{3 / 2,1 / 2,2}(x)=\frac{3}{8}(1-x)^{3 / 2}(1+x)^{1 / 2}, \\
& \hat{P}_{2+k}^{(3 / 2,1 / 2,2)}=\frac{3}{8} P_{k}^{(3 / 2,1 / 2)}, \quad k \geq 0 .
\end{aligned}
$$

In other words, for certain singular values of the parameters, the codimension is actually less than $m$, and in some instances (such as the one above) even yield the classical polynomials. The condition (102) must be imposed in order to avoid such singular possibilities.

Proposition 4.6. Suppose that $\alpha, \beta>-1$ satisfy the conditions of Propositions 4.4 and 4.5 The $L^{2}$ norms of the $X_{m}$ Jacobi polynomials are given by

$\int_{-1}^{1}\left[\hat{P}_{m+k}^{(\alpha, \beta, m)}(x)\right]^{2} \hat{W}_{\alpha, \beta, m} d x=\frac{(1+\alpha+k-m)(\beta+m+k)}{(\alpha+1+k)^{2}} N_{k}^{\alpha+1, \beta-1}, k \geq 0$.

Proof. This follows directly from (59).

We summarize the above findings as follows.

Theorem 4.1. Let $m>1$ and $\alpha, \beta>-1$ be such that $\alpha>m-$ $2, \operatorname{sgn}(\alpha-m+1)=\operatorname{sgn}(\beta)$ and such that (102) holds. Let $\mathcal{U}$ be the stable, codimension $m$ flag spanned by polynomials $y(x)$ such that $(1+x) y^{\prime}+\beta y$ is divisible by $P_{m}^{(-\alpha-1, \beta-1)}(x)$. Let $\hat{W}_{\alpha, \beta, m}(x)$ be the weight defined by (54). Then, the $X_{m}$ Jacobi polynomials, as defined by (94) are the orthogonal polynomials obtained by orthogonalizing the flag $\mathcal{U}$ relative to the weight $\hat{W}_{\alpha, \beta, m}(x)$ and subject to the normalization condition (98).

Finally, let us discuss shape-invariance of the generalized Jacobi operators. The following Proposition was proved in [22].

Proposition 4.7. Let $\hat{A}, \hat{B}$ be the operators defined in (108) (109). Then

$$
T_{\alpha, \beta, m}=\hat{B}_{\alpha, \beta, m} \hat{A}_{\alpha, \beta, m}, \quad \hat{A}_{\alpha, \beta, m} \hat{P}_{m}^{(\alpha, \beta, m)}=0
$$

is the state-deleting factorization of the $X_{m}$ Jacobi operator. Furthermore,

$$
T_{\alpha+1, \beta+1, m}=\hat{A}_{\alpha, \beta, m} \hat{B}_{\alpha, \beta, m}+\alpha+\beta+2,
$$

is the dual state-adding factorization.

In essence, we are asserting that the generalized operators obey the same shape-invariance relations as their classical counterparts; c.f., equations (73) (74). 
The proof of Proposition 4.7 relies on the permutability property of higher order Darboux transformation and goes beyond the scope of this survey. We limit ourselves to explicitly deriving the raising and lowering operators used in the above factorization.

We already know the factorization eigenfunction:

$$
\phi(x)=\hat{P}_{m}^{(\alpha, \beta, m)}(x)=(-1)^{m} \frac{1+a-m}{1+a} P_{m}^{(-\alpha-2, \beta)}(x) .
$$

We make use of (69) to determine the factorization gauge. Making use of the fact that $h(\alpha, \beta)=(\alpha+1, \beta+1)$ we obtain

$$
b(x)=\frac{\xi_{\alpha+2, \beta, m}}{\xi_{\alpha+1, \beta-1, m}} .
$$

We use Proposition 4.2 to derive the dual factorization gauge

$$
\hat{b}(x)=\left(1-x^{2}\right) \frac{\xi_{\alpha+1, \beta-1, m}}{\xi_{\alpha+2, \beta, m}}
$$

and the dual factorization eigenfunction,

$$
\hat{\phi}(x)=(1-x)^{-\alpha-1}(1+x)^{-\beta-1} \xi_{\alpha+1, \beta-1, m} .
$$

We thereby obtain

$$
\begin{aligned}
& \hat{A}_{\alpha, \beta, m}(y)=\frac{\xi_{\alpha+2, \beta, m}}{\xi_{\alpha+1, \beta-1, m}}\left(y^{\prime}-\rho_{\alpha+2, \beta, m} y\right) \\
& \hat{B}_{\alpha, \beta, m}(y)=\left(1-x^{2}\right) \frac{\xi_{\alpha+1, \beta-1, m}}{\xi_{\alpha+2, \beta, m}}\left[y^{\prime}-\left(\rho_{\alpha+1, \beta-1, m}+\frac{\alpha+1}{1-x}-\frac{\beta+1}{1+x}\right) y\right]
\end{aligned}
$$

These shape-invariant factorizations serve as a good illustration of the duality between formal state-adding and state-deleting transformations; here $\phi(x)$ is a polynomial but $\hat{\phi}(x)$ is merely a quasi-rational function.

As well, the shape-invariant factorization illustrates that $b(x)$, the factorization gauge, is not necessarily a polynomial. Here,

$$
w=-\phi^{\prime} / \phi=\rho_{\alpha+2, \beta, m} .
$$

The denominator is $\xi_{\alpha+2, \beta, m}$ but the transformation

$$
y \mapsto \xi_{\alpha+2, \beta, m}\left(y^{\prime}-w y\right), \quad y=\hat{P}_{n}^{(\alpha, \beta, m)}, n \geq m
$$

would produce an imprimitive flag. The common factor is $\xi_{\alpha+1, \beta-1, m}$, and that is why the correct factorization gauge is the rational function shown in (107). 
As a consequence of these shape-invariant factorizations, we have the following lowering and raising identities for the $X_{m}$ Jacobi operators; c.f., (78) (79)

$$
\begin{aligned}
& \hat{B}_{\alpha, \beta, m} \hat{P}_{m+k}^{(\alpha+1, \beta+1, m)}=2(1+k) \hat{P}_{m+k+1}^{(\alpha, \beta, m)}, \quad k \geq 0 ; \\
& \hat{A}_{\alpha, \beta, m} \hat{P}_{m+k}^{(\alpha, \beta, m)}=\frac{1}{2}(1+\alpha+\beta+k) \hat{P}_{m+k-1}^{(\alpha+1, \beta+1, m)} .
\end{aligned}
$$

Acknowledgements. We thank Ferenc Tookos for useful comments and suggestions. The research of DGU was supported in part by MICINN-FEDER grant MTM2009-06973 and CUR-DIUE grant 2009SGR859. The research of NK was supported in part by NSERC grant RGPIN 105490-2004. The research of RM was supported in part by NSERC grant RGPIN-228057-2004.

\section{REFERENCES}

[1] J. Aczel, Eine Bemerkung über die Charakterisierung der klassichen orthogonale Polynome, Acta Math. Acad.Sci. Hungar 4 (1953), 315-321.

[2] R.A. Askey and J.A. Wilson, Some basic hypergeometric orthogonal polynomials that generalize Jacobi polynomials, Memoirs American Mathematical Society No. 3191985.

[3] F.V. Atkinson and W.N. Everitt, Orthogonal polynomials which satisfy second order differential equations. E. B. Christoffel (Aachen/Monschau, 1979), pp. 173-181, Birkhäuser, Basel-Boston, Mass., 1981.

[4] Erdélyi A et al. Higher Transcendental Functions, Vol. I, McGraw-Hill, New York, 1953

[5] S. Bochner, Über Strum-Liouvillesche Polynomsysteme, Math. Z. 29 (1929), 730-736.

[6] F. Cooper, A. Khare and U. Sukhatme, Supersymmetry in quantum mechanics World Scientific, Singapore 2001

[7] P. Crooks and R. Milson, On projective equivalence of univariate polynomial subspaces, SIGMA 5 (2009), paper 107

[8] P. A. Deift, Applications of a commutation formula, Duke Math. J. 45 (1978), $267-310$

[9] S. Y. Dubov, V. M. Eleonskii, and N. E. Kulagin, Equidistant spectra of anharmonic oscillators, Sov. Phys.JETP 75 (1992) 47-53

[10] W. N. Everitt, K. H. Kwon, L. L. Littlejohn and R. Wellman, Orthogonal polynomial solutions of linear ordinary differential equations, J. Comput. Appl. Math 133 (2001), 85-109.

[11] W. N. Everitt, L. L. Littlejohn, R. Wellman, The Sobolev orthogonality and spectral analysis of the Laguerre polynomials $L_{n}^{-k}$ for positive integers $k, J$. Comput. Appl. Math. 171 (2004), 199-234.

[12] J. Feldmann, On a characterization of classical orthogonal polynomials, Acta Sc. Math. 17 (1956), 129-133.

[13] L.E. Gendenshtein, Derivation of exact spectra of the Schrodinger equation by means of supersymmetry, JETP Lett. 38 (1983) 356-359 
[14] F. Gesztesy and G. Teschl, On the double commutation method, Proc. Amer. Math. Soc. 124 1831-1840 (1996)

[15] J. Gibbons and A.P. Veselov, On the rational monodromy-free potentials with sextic growth, J. Math. Phys. (50) (2009) 013513, 25 pages.

[16] D. Gómez-Ullate, N. Kamran and R. Milson, The Darboux transformation and algebraic deformations of shape-invariant potentials, J. Phys. A 37 (2004), 1789-1804.

[17] D. Gómez-Ullate, N. Kamran and R. Milson, Supersymmetry and algebraic Darboux transformations, J. Phys. A 37 (2004), 10065-10078.

[18] D. Gómez-Ullate, N. Kamran and R. Milson, Quasi-exact solvability and the direct approach to invariant subspaces. J. Phys. A, 38(9):2005-2019, 2005.

[19] D. Gómez-Ullate, N. Kamran and R. Milson, Quasi-exact solvability in a general polynomial setting, Inverse Problems, 23 (2007) 1915-1942.

[20] D. Gómez-Ullate, N. Kamran, and R. Milson, An extension of Bochner's problem: exceptional invariant subspaces J. Approx. Theory 162 (2010) 987-1006.

[21] D. Gómez-Ullate, N. Kamran, and R. Milson, An extended class of orthogonal polynomials defined by a Sturm-Liouville problem, J. Math. Anal. Appl. 359 (2009) 352-367.

[22] D. Gómez-Ullate, N. Kamran, and R. Milson, Exceptional orthogonal polynomials and the Darboux transformation, J. Phys. A 43 (2010) 434016

[23] E. Hendriksen and H. van Rossum, Semiclassical orthogonal polynomials, in "Orthogonal polynomials and applications" (Bar-le-Duc, 1984), C. Brezinski et al Editors, 354-361, Lecture Notes in Math., 1171, Springer, Berlin, 1985.

[24] M. Ismail, Classical and quantum orthogonal polynomials in one variable, Encyclopedia in Mathematics, vol 98 Cambridge University Press, Cambridge, (2005).

[25] R. Koekoek, P. Lesky, and R. Swarttouw, Hypergeometric orthogonal polynomials and their q-analogues, Springer-Verlag, Berlin, 2010

[26] K. H. Kwon and L. L. Littlejohn, Classification of classical orthogonal polynomials, J. Korean Math. Soc. 34 (1997), 973-1008.

[27] P. Lesky, Die Charakterisierung der klassischen orthogonalen Polynome durch Sturm-Liouvillesche Differentialgleichungen, Arch. Rat. Mech. Anal. 10 (1962), 341-352.

[28] B. Midya and B. Roy, Exceptional orthogonal polynomials and exactly solvable potentials in position dependent mass Schrödinger Hamiltonians, Phys. Lett. A 373(45) (2009) 4117-4122

[29] M. Mikolás, Common characterization of the Jacobi, Laguerre and Hermitelike polynomials(in Hungarian), Mate. Lapok 7 (1956), 238-248.

[30] C. Quesne, Exceptional orthogonal polynomials, exactly solvable potentials and supersymmetry, J. Phys. A 41 (2008) 392001-392007

[31] C. Quesne, Solvable rational potentials and exceptional orthogonal polynomials in supersymmetric quantum mechanics, SIGMA 5 (2009), 084, 24 pages.

[32] M. Reed and B. Simon, Methods of Modern Mathematical Physics. II. Fourier Analysis, Self-Adjointness, Academic Press, New York-London, 1975.

[33] A. Ronveaux, Sur l'équation différentielle du second ordre satisfaite par une classe de polynômes orthogonaux semi-classiques, C. R. Acad. Sci. Paris Sér. I Math. 305 (1987), no. 5, 163-166. 
[34] A. Ronveaux and F. Marcellán, Differential equation for classical-type orthogonal polynomials, Canad. Math. Bull. 32 (1989), no. 4, 404-411.

[35] S. Odake and R. Sasaki, Infinitely many shape invariant potentials and new orthogonal polynomials, Phys. Lett. B 679 (2009) 414-417.

[36] S. Odake and R. Sasaki, Infinitely many shape invariant discrete quantum mechanical systems and new exceptional orthogonal polynomials related to the Wilson and Askey-Wilson polynomials, Phys. Lett. B 682 (2009), 130-136.

[37] S. Odake and R. Sasaki, Infinitely many shape invariant potentials and cubic identities of the Laguerre and Jacobi polynomials, J. Math. Phys. 51 (2010), 053513, 9 pages.

[38] S. Odake and R. Sasaki, Another set of infinitely many exceptional $\left(X_{\ell}\right)$ Laguerre polynomials, Phys. Lett. B 684 (2010), 173-176.

[39] R. Sasaki, S. Tsujimoto, and A. Zhedanov, Exceptional Laguerre and Jacobi polynomials and the corresponding potentials through Darboux-Crum transformations, J. Phys. A 43 (2010), 315204, 20 pages

[40] C. V. Sukumar, Supersymmetric quantum mechanics of one-dimensional systems, J. Phys. A 18 (1985) 2917-2936.

[41] G. Szegö, Orthogonal polynomials, Amer. Math. Soc. Colloq. Publ. 23, Amer. Math. Soc., Providence RI, 1975, Fourth Edition.

[42] T. Tanaka, $N$-fold Supersymmetry and quasi-solvability associated with $X_{2}$ Laguerre polynomials, arXiv:0910.0328 [math-ph]

[43] V. B. Uvarov, The connection between systems of polynomials that are orthogonal with respect to different distribution functions, USSR Computat. Math. and Math. Phys. 9 (1969), 25-36.

[44] A. Zettl, Sturm-Liouville theory, Mathematical Surveys and Monographs, 121, Amer. Math. Soc., Providence, RI, 2005

Departamento de Física Teórica II, Universidad Complutense de MADRID, 28040 MADRID, SPAIN

Department of Mathematics and Statistics, McGill University MonTREAL, QC, H3A 2K6, CANADA

Department of Mathematics and Statistics, Dalhousie University, Halifax, NS, B3H 3J5, Canada 